\title{
A hipótese "trabalhador feliz, produtivo": o que pensam os servidores públicos federais
}

\author{
The assumption "happy, productive worker": what the federal civil servants think \\ Natasha Fogaça ${ }^{1}$ \\ Universidade de Brasília / Faculdade de Administração, Contabilidade e Economia, Programa de Pós-Graduação em \\ Administração, Brasília - DF, Brasil
}

Francisco Antônio Coelho Junior ${ }^{2}$

Universidade de Brasília / Faculdade de Administração, Contabilidade e Economia, Departamento de administração, Brasília DF, Brasil

\section{Resumo}

\begin{abstract}
A hipótese "trabalhador feliz, produtivo" afirma que os funcionários felizes, cujas necessidades são satisfeitas em seus locais de trabalho, apresentam maior desempenho que os empregados infelizes. Essa é uma afirmação consolidada pela sociedade empresarial, mas com pouquíssimas referências empíricas. Portanto, o objetivo deste estudo é analisar a percepção dos trabalhadores em relação à hipótese "trabalhador feliz, produtivo". Foi empreendido, em caráter exploratório, um grupo focal para compreender como os participantes entendem as variáveis estudadas. O grupo focal foi realizado em uma organização do Poder Judiciário pertencente à administração pública federal e contou com um grupo composto por 7 pessoas de diversas áreas: 2 gestores e 5 servidores de níveis inferiores. Sintetizando os achados, as evidências obtidas aqui apontam o desenvolvimento de estudos que contribuam para estabelecer uma teoria que sustente empiricamente a relação entre satisfação no trabalho, bem-estar no trabalho, desempenho individual no trabalho e estrutura organizacional e que, dessa forma, possa fundamentar a tão disseminada hipótese "trabalhador feliz, produtivo". Ao final, este estudo também possibilitou levantar algumas hipóteses para estudos quantitativos futuros; por exemplo, o clima organizacional poderá aumentar positivamente a relação entre bem-estar no trabalho e desempenho individual no trabalho e a estrutura física estará positivamente associada com o desempenho individual no trabalho.
\end{abstract}

Palavras-chave: Desempenho individual no trabalho. Bem-estar no trabalho. Satisfação no trabalho. Estrutura organizacional. Grupo focal.

\section{Abstract}

The assumption "happy, productive worker" claims that happy employees, whose needs are met at their workplaces, show a better performance than unhappy employees. This is a consolidated statement for the business society, but there are very few empirical references. Therefore, this study aims to examine workers' perception in relation to the assumption "happy, productive worker". A focus group was conducted, having an exploratory nature, in order to understand how participants see the variables under study. The focus group was conducted in an organization of the

Artigo submetido em 30 de junho de 2014 e aceito para publicação em 6 de março 2015.

DOI: http://dx.doi.org/10.1590/1679-395126953

${ }^{1}$ Mestre em Administração pela Universidade de Brasília (UnB). Endereço: Universidade de Brasília, Faculdade de Administração, Contabilidade e Economia (FACE), Programa de Pós - Graduação em Administração. Campus Darcy Ribeiro, Asa Norte, CEP 70910-900, Brasília - DF, Brasil. E-mail: natasha.adm@gmail.com

${ }^{2}$ Doutor em psicologia social, do trabalho e organizacional pela Universidade de Brasília/UnB; Professor adjunto do Departamento de Administração da Universidade de Brasília; Professor do Programa de Pós-graduação em Administração da Universidade de Brasília. Endereço: Universidade de Brasília, Faculdade de Administração, Contabilidade e Economia (FACE), Programa de Pós Graduação em Administração. Campus Darcy Ribeiro, Asa Norte, CEP 70910-900, Brasília - DF, Brasil. E-mail acoelho@unb.br 
Judiciary that belongs to the federal government and it relied on a group made up of 7 people from various areas: 2 managers and 5 civil servants at lower levels. Summarizing findings, the evidence obtained here points out the development of studies that contribute to establish a theory that empirically supports the relation between job satisfaction, well-being at work, individual performance at work, and organizational structure that, thus, can provide some grounding to the widely spread assumption "happy, productive worker". In the end, this study also allowed raising some hypotheses for further quantitative studies; for instance, the organizational climate can positively enhance the relation between well-being at work and individual performance at work and the physical structure will be positively associated with individual performance at work.

Keywords: Individual performance at work. Well-being at work. Job satisfaction. Organizational structure. Focus group.

\section{Introdução}

As organizações são entidades que fazem parte da vida das pessoas, interferindo, inclusive, em seus pensamentos, sentimentos e ações dentro e fora do ambiente de trabalho. $\mathrm{O}$ inverso também pode ser afirmado, uma vez que os pensamentos, sentimentos e ações das pessoas também afetam as organizações nas quais trabalham. O campo de comportamento organizacional (CO) preocupa-se tanto com a influência da organização sobre o indivíduo quanto com a influência do indivíduo sobre a organização (BRIEF e WEISS, 2002). Isso quer dizer que contempla tanto a influência do aspecto macro sobre o micro (top-down), quanto a influência do micro sobre o macro (bottom-up).

Considerando a perspectiva top-down, é factível que o ambiente externo interfere nas emoções, no humor e no afeto do trabalhador, influenciando, consequentemente, o clima favorável (ou não) ao bem-estar no trabalho. Conforme propõem Gondim e Siqueira (2004), emoções de ansiedade e estresse relatados frequentemente pelos trabalhadores atuais têm sido desencadeadas pelo avanço acelerado no ritmo de produção devido às novas tecnologias na organização de trabalho. As consequências advindas da ansiedade e do estresse podem ser prejudiciais tanto para a saúde do trabalhador quanto para a organização.

Pode-se inferir, com base no trabalho de Gondim e Siqueira (2004), que os estados afetivos presentes nas interações humanas começaram a ganhar espaço na discussão sobre a racionalidade nos experimentos realizados em Hawthorne. O interesse geral foi na relação entre as condições de trabalho e a incidência de fadiga e monotonia entre os funcionários. Ao final, são expressas algumas conclusões sobre as reclamações feitas pelos trabalhadores: 1) a fonte das queixas não é advinda de uma única causa, muitas vezes é um efeito geral decorrente de uma situação complexa; 2) a análise de situações complexas exige uma compreensão da natureza do equilíbrio, ou desequilíbrio; 3) as interferências que ocorrem na indústria podem vir de mudanças no ambiente físico, no ambiente social do trabalho, ou de alterações exógenas, e os desequilíbrios podem ser orgânicos, mentais, ou ambos; 4) a fadiga, a monotonia e a supervisão podem encobrir diferentes tipos de interferências e de desequilíbrios, e; 5) os problemas não têm uma única solução (BURREL e MORGAN, 2001).

A preocupação com as emoções e os afetos envolvidos no trabalho tem sido frequente em estudos recentes, em razão do crescente número de empregados com problemas de saúde. O foco recai, especialmente, na compreensão de como as emoções vivenciadas pelos indivíduos em decorrência do trabalho afetam sua saúde e seu desempenho profissional (GOUVEIA, FONSÊCA, LINS et al., 2008). Para a compreensão de como as emoções são tratadas no ambiente de trabalho, Gondim e Siqueira (2004) partem da premissa de que os contextos sociais buscam normalizar a expressão de emoções, de maneira que as mesmas sejam previsíveis e se ajustem às diversas situações ambientais; e esse tipo de situação que tem despertado cada vez mais o interesse de estudiosos do comportamento organizacional e da administração.

Nas últimas três décadas, a capacitação e a autogestão de equipes, equipes de alto desempenho de trabalho e outras práticas de design têm sido muito defendidas nas organizações. Pode-se afirmar que o interesse recente das organizações em projetos de trabalho parte de uma crença de que certos tipos de projeto de trabalho fornecerão vantagem competitiva, e não do desejo de melhorar o bem-estar do empregado. Mesmo 
assim, pouca atenção tem sido dada à forma como o desempenho no trabalho é realmente afetado pelo projeto de trabalho (PARKER e TURNER, 2002).

Paschoal e Tamayo (2008) expressam que algumas práticas organizacionais estão relacionadas com o bemestar do trabalhador. Por exemplo, a irregularidade no horário de trabalho, e o trabalho temporário impactam negativamente no bem-estar, bem como práticas de incentivo à participação, valorização dos empregados, flexibilidade e hierarquia mais horizontalizada, possibilidades de desenvolvimento pessoal e profissional, e a estrutura oferecida favorecem o bem-estar individual dos funcionários.

Warr (2007) propõe que associações significativas entre felicidade e desempenho podem provavelmente surgir a partir de uma terceira variável no ambiente de trabalho, de tal forma que certos recursos do trabalho podem aumentar a relação entre o bem-estar do funcionário e determinadas atividades de trabalho. Em especial, a evidência empírica apresentada no trabalho de Rego (2009) sugere que o bem-estar afetivo no trabalho explica variância significativa de uma medida de autorrelato para desempenho individual. Os colaboradores com elevados níveis de bem-estar afetivo no trabalho autodescrevem-se como detentores de elevado desempenho individual. Ao final, o autor propõe que pesquisas futuras poderão incluir variáveis moderadoras que permitam explicar as conexões entre emoções positivas e desempenho.

Com base nos pressupostos do movimento das relações humanas, a hipótese "trabalhador feliz, produtivo", afirma que os funcionários felizes, cujas necessidades são satisfeitas em seus locais de trabalho, apresentam maior desempenho, que os empregados infelizes (SONNENTAG, 2002). A conexão existe e é de conhecimento geral, entretanto as organizações não dispõem de dados suficientes para estabelecer as relações de causa e efeito entre bem-estar, satisfação e desempenho no trabalho, e assim, fazer as melhorias para as pessoas e, consequentemente, contribuir para o desempenho organizacional. Tomando essas indicações como base, o objetivo deste estudo é analisar a percepção dos trabalhadores em relação à hipótese "trabalhador feliz, produtivo".

Conforme proposto por Coelho Jr., Gondim, Borges-Andrade e Faiad (2012), comumente as evidências empíricas nos estudos em Comportamento Organizacional são originárias das percepções individuais, mas não são suficientes para a construção de uma teoria geral dos fenômenos organizacionais. Siqueira (2002) aponta, como crítica, que grande parte dos estudos em CO têm sido realizados utilizando como ferramentas de análise apenas medidas de percepção dos empregados sobre as variáveis organizacionais; a autora aponta a necessidade de outras formas de análise das informações, para que não se permaneça apenas nas investigações individuais e situacionais que tradicionalmente vêm sendo conduzidas.

Assim, foi empreendido, em caráter exploratório, um grupo focal para compreender como os participantes entendem as variáveis estudadas. O grupo focal como método de coleta de dados já está legitimado na tradição de pesquisa qualitativa (GRØNKJÆR, CURTIS, CRESPIGNY et al., 2011). Segundo Flick (2009) e Oliveira e Freitas (1998), a obtenção de interpretações por parte dos participantes sobre os resultados obtidos em estudos anteriores e explorar aspectos de análise de dados já coletados são utilidades do grupo focal. Neste trabalho, portanto, a utilização desse método se justifica para que se compreenda como os participantes deste estudo entendem as relações entre as variáveis bem-estar no trabalho, satisfação no trabalho, desempenho individual no trabalho e estrutura organizacional.

O desempenho individual é uma variável-chave em psicologia organizacional, isso se justifica pelo uso generalizado de medidas de desempenho individuais em pesquisas e meta-análises. Do ponto de vista prático, essa premissa faz todo o sentido, isso porque o desempenho individual é algo que as organizações buscam melhorar e otimizar (SONNENTAG e FRESE, 2002).O desempenho é um aspecto primordial para qualquer organização. São os resultados obtidos pela organização que interessam ao gestor, e são esses resultados que determinam o sucesso de qualquer empresa. Deve-se ter em mente que esses resultados gerais são fruto da sinergia dos resultados individuais, de modo que, conhecendo os fatores que propiciam ou favorecem o alcance de resultados positivos, segundo a perspectiva desses indivíduos, é possível desenvolver estratégias ou práticas para que essas pessoas mantenham bons níveis de desempenho. 
A escolha da estrutura organizacional como variável de contexto a ser estudada se deve ao fato de que a relação entre estrutura e fator humano é condição sinequa non para o sucesso de qualquer organização (VASCONCELLOS e HEMSLEY, 2002), de modo que não considerar seus aspectos ou componentes seria o mesmo que negligenciar a importância da estrutura no desempenho do indivíduo. Pode-se dizer que a estrutura é transversal a tudo o que acontece na organização. O conhecimento do papel dos componentes da estrutura possibilitará traçar estratégias e práticas capazes de incrementar a relação estudada. Como pode ser considerada uma importante variável de contexto, e diversos pesquisadores clamam pela inclusão de variáveis de contexto nas relações que envolvem desempenho individual, sua adoção é justificada.

Conforme referenciado em momento anterior, a questão que norteia essa pesquisa foi originada com base na hipótese apresentada por Sonnentag (2002), "trabalhador feliz, produtivo". Assim, para que fosse possível dimensionar a felicidade do trabalhador, recorreu-se a duas variáveis tradicionalmente trabalhadas em comportamento organizacional: "satisfação no trabalho" e "bem-estar no trabalho". A seleção desses dois construtos se deve especialmente à própria definição do termo felicidade, apresentada no dicionário Houaiss da seguinte maneira: "qualidade ou estado de feliz; estado de uma consciência plenamente satisfeita; satisfação, contentamento, bem-estar".

Espera-se, portanto, que os resultados obtidos com este trabalho possam impulsionar o desenvolvimento de estudos futuros sobre essa temática; além disso, embasar a construção de estratégias ou práticas organizacionais na organização estudada, que propiciem maior bem-estar e satisfação dos funcionários e, ao mesmo tempo, incrementem o desempenho dos indivíduos e facilitem o alcance dos objetivos organizacionais com maior efetividade.

\section{Referencial Teórico}

No campo da Psicologia Organizacional e do Trabalho, o conceito de desempenho individual é um conceito central (SONNENTAG e FRESE, 2002). Apesar de sua fundamental importância em quase todas as decisões que envolvem os recursos humanos, destaca-se o fato de que ainda não existe uma teoria abrangente sobre desempenho no trabalho (CAMPBELL, 1990; DEADRICK e GARDNER, 2008). Contudo, nos últimos 15 anos têm sido feitos progressos na clarificação e no alargamento do conceito de desempenho (CAMPBELL, 1990). Os avanços se concentraram principalmente na especificação de preditores e processos associados com o desempenho individual (SONNENTAG e FRESE, 2002).Sonnentag e Frese (2002) expressam que desempenho representa as habilidades e as competências aplicadas no contexto do cargo à execução de uma obrigação ou tarefa, ou a maneira como atua um indivíduo em termos de eficiência e rendimento, culminando no alcance de resultados organizacionais. Refere-se, portanto, ao empreendimento intencional pelo indivíduo em uma ação que é orientada pelo seu resultado, isto é, uma ação que tem um propósito consciente ou motivação previa.

De acordo com Chase, Jacobs e Aquilano (2006) e Dejours (2008), o desempenho humano pode ser influenciado por fatores comportamentais ou carga mental do trabalho (por exemplo, a motivação e a estrutura do grupo de trabalho) e pela carga física decorrente do projeto de trabalho. Oliveira-Castro, Lima e Veiga (1996) consideram que o desempenho humano no trabalho é consequência das competências individuais inerentes a cada trabalhador, das relações interpessoais, do ambiente e das características da organização.

As principais pesquisas na área de bem-estar no trabalho referem-se ao bem-estar geral, desvinculado de qualquer contexto. Pesquisas têm revelado a influência de fatores organizacionais ou de percepções de aspectos organizacionais sobre o bem-estar do indivíduo (PASCHOAL e TAMAYO, 2008). Segundo Gouveia et al. (2008), o mínimo necessário para desenvolver as atividades laborais com eficácia e prazer apoia-se no bem-estar. 
Evidências teóricas e empíricas sugerem que o fomento do bem-estar psicológico parece ser uma boa maneira de promover o sucesso e o desempenho individual e organizacional (WRIGHT e CROPANZANO, 2000). Existem trabalhos em que as pessoas sofrem pressões, convivem com constantes situações de fracasso e ameaças de desemprego. Nestes casos, as necessidades dos indivíduos não são satisfeitas, havendo um aumento progressivo de emoções negativas chegando ao ponto de os trabalhadores perderem a satisfação com o que fazem e não gozarem de bem-estar. (KIRSCHENBAUM, OIEGENBLICK e GOLDBERG, 2000). Parece evidente, pois, ter em conta que o bem-estar pode ser útil para estimar o seu desempenho e potencial envolvimento em acidentes laborais. O tempo dedicado ao trabalho constitui um componente fundamental para a construção e o desenvolvimento do bem-estar pessoal e da felicidade (VAN HORN, TARIS, SHAUFELI et al., 2004).

O bem-estar no trabalho consiste em um dos fenômenos relacionados a experiências positivas que vem se destacando na literatura organizacional internacional nos últimos anos. No Brasil, por sua vez, existe um vasto campo a ser explorado e compreendido por pesquisadores e gestores organizacionais (PASCHOAL, DEMO, FOGAÇA et al., 2013). Normalmente, as pesquisas empreendidas sobre bem-estar, incluindo a literatura científica nacional, enfocam o bem-estar geral, aquele desvinculado de qualquer contexto, e fenômenos apenas relacionados ao bem-estar no trabalho, como estresse ocupacional e burnout (PASCHOAL, 2008).Seguindo a linha de Warr (2007), Paschoal e Tamayo (2008) apontam que bem-estar no trabalho é a prevalência de emoções positivas no trabalho e a percepção do indivíduo de que, no seu atuar, expressa e desenvolve seus potenciais e avança no alcance de suas metas de vida. O bem-estar no trabalho, deste modo, inclui tanto aspectos afetivos (emoções e humores) quanto cognitivos (percepção de realização).

A revisão feita por Sonnentag (2002) dos estudos sobre a relação entre bem-estar individual e desempenho no trabalho demonstrou que aspectos específicos de bem-estar estão positivamente relacionados ao desempenho no trabalho, de modo que esses achados sugeriram que uma melhora no nível de bem-estar do indivíduo gera uma melhora no desempenho no trabalho do indivíduo. O objetivo do trabalho de Rego (2009) foi demonstrar empiricamente a relação entre bem-estar afetivo no trabalho e desempenho individual, utilizando escalas de autorrelato. Os resultados obtidos nessa pesquisa apresentaram que o bem-estar afetivo no trabalho explica $23 \%$ da variância significativa de uma medida de desempenho individual. Com base nesse resultado, pode-se inferir que pessoas com maiores níveis de bem-estar afetivo no trabalho afirmaram possuir elevado desempenho individual. Contudo, o autor alerta para ter cautela com os resultados encontrados, uma vez que a amostra utilizada não era expressiva, podendo ser questionada do ponto de vista estatístico; sugeriu, portanto, que estudos futuros contassem com amostra de maior dimensão.

Também preocupados em entender a relação entre bem-estar psicológico e desempenho no trabalho, Wright e Cropanzano (2000) revistaram alguns estudos feitos sobre a relação realizados nos anos de 1993 e 1997 , pelo primeiro autor. No primeiro estudo, verificou-se que bem-estar se relacionou positivamente com avaliações de desempenho feitas por supervisores. O segundo estudo apresentado demonstrou uma relação significativa entre bem-estar e desempenho no trabalho. No trabalho em questão, os autores se propõem a realizar um teste comparativo da contribuição relativa da satisfação no trabalho e bem-estar psicológico como preditores de desempenho do trabalhador. As duas análises realizadas no teste demonstraram a contribuição relativa de bem-estar na predição de desempenho, verificando que o bem-estar foi relacionado a avaliações de desempenho para além do efeito da satisfação no trabalho.

A produção recente aponta a tendência de trabalhar com uma perspectiva negativa do bem-estar, isto é, as relações estudadas enfocam a exaustão emocional, por exemplo. Contudo, considerar os resultados dessas pesquisas são de suma importância, tendo em vista que foram encontradas evidências de que a exaustão emocional se relaciona negativamente com desempenho no trabalho (CROPANZANO, RUPP e BYRNE, 2003; HALBESLEBEN e BOWLER, 2007; JANSSEN, LAM e HUANG, 2010). É importante destacar, ainda, que estudos que envolveram as relações entre bem-estar no trabalho e desempenho no trabalho, como em Kaplan, Bradley, Luchman et al. (2009) e Wright e Huang (2012), apontaram a existência de uma relação preditiva do bem-estar em relação ao desempenho, em consonância com os estudos apresentados anteriormente (REGO, 2009; SONNENTAG, 2002; WRIGHT e CROPANZANO, 2000). 
Um dos conceitos mais largamente discutidos e pesquisados em relatos empíricos em psicologia organizacional, comportamento organizacional e gestão de pessoas é a satisfação no trabalho (TSANG e WONG, 2005; WORRELL, SKAGGS e BROWN, 2006). Desde 1980, a satisfação no trabalho tem sido investigada como uma importante variável independente em pesquisas de comportamento organizacional, o que não impediu que também fossem desenvolvidos trabalhos que consideraram os efeitos de variáveis antecedentes na satisfação do trabalhador (CHANG e LEE, 2006).Satisfação no trabalho, de acordo com Siqueira (2008), consiste na totalização do quanto o indivíduo que trabalha vivencia experiências prazerosas no contexto das organizações e é mensurada pelo grau de contentamento com a natureza do trabalho, com os colegas do trabalho, com promoções, com a chefia e com o salário.

A literatura em comportamento organizacional apresenta um grande número de estudos empíricos acerca das relações preditivas entre satisfação no trabalho e desempenho (BRIEF e WEISS, 2002; COELHO JR., 2009; TSANG e WONG, 2005). Segundo Wright e Cropanzano (2000),há importantes evidências empíricas que sinalizam a existência de correlações entre as variáveis satisfação e desempenho no trabalho. Autores como Bates (1999), Bretz Jr., Milkovich e Read (1992) e Coelho Jr. (2009) sugerem, entretanto, que esta relação ainda precisa ser mais bem investigada e aprofundada, especialmente em termos de uma melhor adequação e definição dos instrumentos e medidas, e diversificando-se tanto o método de análise de dados quanto o nível de algumas variáveis investigadas nos modelos teóricos hipotetizados. Isto poderia promover maior predição da variância de desempenho no trabalho e, portanto, maior compreensão teórico/prática deste construto.

O trabalho de Coelho Jr. (2009) demonstrou que satisfação exerce influência no desempenho individual; contudo, essa influência depende da unidade em que o indivíduo esteja lotado. Na revisão da literatura recente, os estudos apontaram para uma relação preditiva positiva entre satisfação no trabalho e desempenho no trabalho, com ênfase, em boa parte dos estudos, no desempenho da tarefa (COELHO JR. e BORGESANDRADE, 2011; EDWARDS, BELL, ARTHUR Jr. et al., 2008; FISCHER, 2003; NETEMEYER, MAXHAM III e LICHTENSTEIN, 2010; SCHLEICHER, WATT e GREGURAS, 2004; SY, TRAM e O'HARA, 2006).

Em contraponto, a pesquisa comparativa desenvolvida por Wright e Cropanzano (2000) verificou que satisfação no trabalho não oferece uma contribuição relativa na predição de desempenho no trabalho; no entanto, os autores alertam que esses resultados podem ter sido afetados pelo tamanho modesto da amostra utilizada, apontando a necessidade de novas pesquisas, uma vez que tradicionalmente satisfação tem sido associada a desempenho no trabalho. O estudo de Bowling (2007), com base em uma meta-análise, destaca que a relação entre satisfação no trabalho e desempenho no trabalho não é relevante, de modo que variáveis como traços de personalidade podem minimizar a intensidade dessa relação. Depreende-se, portanto, que os achados desses autores sinalizam uma relação preditiva entre satisfação e desempenho, mas não significativa por influência de outros aspectos, traduzindo-se em um convite para mais pesquisas que envolvam outras variáveis mediadoras ou moderadoras nessa relação e na utilização de amostras representativas. Altos níveis de desempenho no trabalho e satisfação no trabalho ocorrem quando existe congruência entre as necessidades individuais e as características do trabalho (GORIS, VAUGHT e PETTIT JR., 2000).

A estrutura organizacional consiste em um arcabouço invisível que viabiliza o funcionamento adequado, coordenado, equilibrado e integrado da organização, apresentando as diversas inter-relações existentes entre os diferentes elementos que a constituem. Esse arcabouço estabelece o status e define a posição de cada funcionário em relação aos demais integrantes da organização, especificando quem exerce autoridade sobre quem e com que objetivo. A estrutura da organização é resultado da formulação racional de todos os segmentos presentes na atividade da instituição. O propósito do arcabouço invisível é promover a coordenação dos membros do grupo, de modo que as determinações de autoridade e de responsabilidade estabelecem o que cada pessoa desempenhará dentro de relações apropriadas em relação às demais, no contexto do grupo. Em suma, a estrutura organizacional oferece um modelo sistematizado em relação ao trabalho a ser feito, compreendendo aspectos técnicos e comportamentais, baseados nos condicionantes e componentes específicos que influenciam o seu próprio desenho (GONÇALVES, 1998; OLIVARES, 2003). Oliveira (2006) descreve a estrutura organizacional como o conjunto formado pela especificação das 
responsabilidades dos níveis hierárquicos, pela distribuição de autoridade, pelo sistema de comunicação e pelas decisões das unidades organizacionais.

O trabalho de Siqueira e Amaral (2006) estabelece correlações entre dimensões de estrutura organizacional e dimensões de bem-estar psicológico. Os resultados expressam que o bem-estar psicológico, no que tange às avaliações de autovalor, pode ser comprometido quando os profissionais atuam em empresas cuja distribuição de autoridade tende a ser centralizada e as atividades realizadas rigidamente divididas em unidades organizacionais. E sensações negativas tais como irritação, desânimo, depressão, angústia, nervosismo e chateação podem ser desencadeados no ambiente organizacional por percepções de que existe uma rigidez de autoridade no sistema de comando empresarial.

Os achados do estudo empreendido por Campbell, Fowles e Weber (2004) sugerem que ambientes de trabalho em que superiores consultam subordinados, e vice-versa, sobre tarefas de trabalho e decisões, e em que os indivíduos estão envolvidos com os colegas na tomada de decisão e definição de tarefas, estão positivamente relacionados com a satisfação no trabalho.

Diversos estudos demonstraram o impacto da autonomia nas variáveis bem-estar, satisfação e desempenho no trabalho (DOEST e DE JONGE, 2006; LANGFRED, 2005; LANGFRED e MOYE, 2004; LASCHINGER, FINEGAN, SHAMIAN et al., 2004; LEACH, WALL e ROGELBERG, 2005; MIERLO, RUTTE, VERMUNT et al., 2007; MORGESON, DELANEY-KLINGER e HEMINGWAY, 2005; OZER, 2011; STEWART, 2006), demonstrando a importância desse componente da estrutura organizacional. Em visita feita à literatura recente, infelizmente não foram encontrados estudos que tratassem de todos os componentes da estrutura organizacional, mas os componentes estudados - feedback (ANSEEL e LIEVENS, 2007; LADEIRA, SONZA e BERTE, 2012), autoridade (LIN, WANG e CHEN, 2013); formalização, tomada de decisão, centralização (ARYEE, CHEN e BUDHWAR, 2004) - sinalizam evidências de que a estrutura organizacional exerce influência sobre as variáveis de interesse deste estudo: bem-estar no trabalho, satisfação no trabalho e desempenho.

É evidente que a estrutura organizacional impacta de alguma forma nas emoções e nos afetos do trabalhador; percebe-se, sobretudo, que alguns fatores ou componentes foram mais estudados que outros. Do ponto de vista gerencial, é fundamental contemplar a estrutura em estudos organizacionais, de tal sorte que seja possível identificar seus impactos e, assim, promover adaptações ou mudanças que sejam benéficas tanto para a organização quanto para as pessoas que nela trabalham. Não obstante, a necessidade de estudos que abarquem variáveis contextuais nas relações envolvendo desempenho foi determinante para inclusão dessa variável nesta pesquisa, bem como o fato de que a estrutura é um fator transversal a tudo o que acontece na organização.

\section{Método}

Nesta seção está relacionado o método que será adotado na pesquisa, indicando informações referentes aos participantes da pesquisa e aos procedimentos de coleta e análise dos dados. Este estudo tem caráter descritivo, pois o objetivo aqui é descrever como se manifesta determinado fenômeno (SAMPIERI, COLLADO e LUCIO, 2006). A pesquisa também tem caráter transversal, uma vez que as informações serão coletadas em um único momento da realidade. Será utilizada a técnica de grupo focal como forma de coleta de dados e a técnica de análise de conteúdo para compreender as respostas dos questionários.

\section{Grupo focal}

O objetivo do grupo focal é obter o entendimento dos participantes sobre o tópico de interesse da pesquisa, não importando se é utilizado sozinho ou em conjunto com outros métodos, nem mesmo se busca questões ou respostas (OLIVEIRA e FREITAS, 1998). A lógica da aplicação do método de grupo focal consiste na 
criação do conhecimento com base nas diversas experiências e formas de conhecimento e interação entre os participantes. A diversidade dos participantes e a interação do grupo é favorável para uma maior comunicação entre as pessoas e proporciona a geração de diferentes conteúdos, pois destaca as percepções, atitudes, pensamentos e valores culturais dos participantes; esses fatores em conjunto constituem-se, portanto, na vantagem desse método (GRØNKJÆR, CURTIS, CRESPIGNY et al., 2011).

Contudo, apesar de a interação ser o carro-chefe do grupo focal, esse comportamento raramente tem sido analisado ou discutido em pesquisas empíricas, sendo tratado apenas como um recurso para a coleta de dados. Muitas vezes, os resultados são relatados utilizando as citações de cada indivíduo, dando a impressão de que pontos de vista individuais podem ser isolados. É importante destacar que o papel do moderador é de suma importância para o sucesso da interação, pois o despreparo na sua condução pode interferir nos resultados, em decorrência de uma coleta de má qualidade (GRØNKJÆR, CURTIS, CRESPIGNY et al., 2011). Para Oliveira e Freitas (1998), o envolvimento do moderador possui relação com o objetivo da pesquisa: um baixo nível de envolvimento é indicado quando há ênfase na pesquisa exploratória e na análise de conteúdo; por sua vez, um alto nível de envolvimento é recomendado quando há uma forte agenda a ser cumprida e há a necessidade de comparar o pensamento de grupos de participantes com o que foi obtido em sessões prévias.

\section{Participantes do estudo}

Trata-se de um estudo com amostra intencional realizado em uma organização do poder judiciário pertencente à administração pública federal. Essa organização foi escolhida devido ao seu interesse em participar da presente pesquisa. O objetivo inicial foi contar com participantes de diversas áreas e funções para assegurar a heterogeneidade do grupo focal. Devido às demandas da organização, foi possível contar com a participação de uma secretaria, a secretaria de comunicação, composta por quatro coordenações, totalizando 61 funcionários.

A escolha dos participantes se deu por sorteio feito no dia da realização do grupo focal, para evitar que os participantes preparassem respostas que julgassem adequadas. Para a realização do sorteio, os colaboradores foram separados previamente em 2 grupos, de acordo com sua posição hierárquica, e para assegurar a heterogeneidade do grupo focal. O grupo final foi composto por sete pessoas: dois gestores e cinco servidores, sendo quatro do sexo masculino e três, do sexo feminino. O agrupamento de gestores e servidores foi possível graças à cultura da organização, que promove uma relação mais próxima dos gestores com as equipes, sendo definida por alguns como "familiar"; destaca-se que os servidores são incluídos em alguns processos decisórios, tendo uma participação bem atuante. Além disso, antes de iniciar, foi pedido aos participantes que as discussões do grupo fossem gravadas, o que foi prontamente autorizado.

Com base nas premissas para a realização do grupo focal, buscou-se o consenso nas respostas dos participantes, e, justamente, para o alcance desse objetivo, o grupo teve duração aproximada de duas horas.

\section{Procedimentos de coleta de dados}

A coleta de dados foi feita com base em um roteiro semiestruturado, no qual foram aproveitados todos os conteúdos mencionados pelos entrevistados de maneira espontânea. O roteiro foi composto por perguntas abertas, em um total de 11 questões elaboradas com base no referencial teórico e na hipótese "trabalhador feliz, produtivo".

Em um primeiro momento, pediu-se aos entrevistados que relatassem como definem e compreendem três variáveis deste estudo - satisfação no trabalho, bem-estar no trabalho e componentes da estrutura organizacional. Somente após a descrição do método eram feitos questionamentos sobre como o grupo entende a relação entre as variáveis, de que modo eles observam as influências - se é que elas existem - de uma variável em outra. Assim, as questões elaboradas nessa etapa foram as seguintes: 1) Pensando na estrutura, que fatores colaboram para o seu desempenho no trabalho? E que fatores dificultam? 2) Que 
fatores da estrutura contribuem para o seu bem-estar no trabalho? E quais diminuem o seu bem-estar? 3) Na sua opinião, como tem sido seu desempenho no trabalho quando vivencia situações de bem-estar no trabalho? Melhora, diminui, ou nada acontece? 4) Que fatores da estrutura contribuem para a sua satisfação no trabalho? E quais diminuem sua satisfação? 5) Na sua opinião, como tem sido seu desempenho no trabalho quando satisfeito com seu trabalho? Melhora, diminui, ou nada acontece?

\section{Procedimentos de análise de dados}

Para a análise dos dados obtidos com o grupo focal, foi realizada a análise de conteúdo. Esse método tem como base a análise do conteúdo obtido com grupo focal ou com entrevista, isto é, considera a posição das pessoas quanto aos aspectos pesquisados (BARDIN, 2009). Para a realização dessa análise, foram definidas categorias ex ante com base nas questões; assim, as informações foram divididas em atributos vinculados a cada uma das variáveis, viabilizando a compreensão de como as variáveis são entendidas pelos participantes. Com base nas informações obtidas, foi possível estabelecer subcategorias de análise, amparadas pelas questões que relacionam as variáveis. A partir dessas subcategorias foram determinados os tópicos em que serão apresentados os resultados, dispostos na próxima seção.

\section{Resultados}

As informações obtidas com o grupo focal possibilitaram traçar um panorama sobre as relações das variáveis-objeto deste estudo considerando a perspectiva do trabalhador. Em nenhum momento procurou-se generalizar as proposições feitas. Diante do exposto, os resultados apresentados a seguir enfatizam como os participantes visualizam as relações entre satisfação, bem-estar, desempenho no trabalho e estrutura organizacional em sua realidade profissional.

\section{Componentes da estrutura organizacional}

Em relação à estrutura organizacional, verificou-se que os participantes associam o conceito à estrutura física da organização. Esse entendimento ficou evidente quando foram feitas descrições sobre a qualidade dos equipamentos, da rede de informática e do mobiliário. Sobre os equipamentos e o mobiliário, foram feitos comentários positivos e elogios, de modo que a qualidade dos monitores foi algo bastante frisado, haja vista se tratar de uma secretaria de comunicação. O conforto das cadeiras e a preocupação com a ergonomia também foi um fator bastante ressaltado. Por sua vez, a rede de informática foi bastante criticada, pois determinados recursos e programas necessários para as atividades da secretaria travam com facilidade, e arquivos são bloqueados durante os procedimentos de backup. Contudo, a equipe de assessoria técnica em informática foi bastante elogiada por sua presteza em atender às solicitações feitas.

Dado que o entendimento de estrutura pelos participantes se restringia aos aspectos físicos, durante a discussão foram propostas questões dirigidas aos quatro componentes da estrutura organizacional, conforme a definição de Oliveira (2006): sistema de responsabilidade, sistema de autoridade, sistema de comunicação e sistema de decisão. A comunicação foi primeiro fator trabalhado. Destaca-se que a comunicação interna na secretaria em questão flui bem, o que possibilita uma forte integração entre as coordenações e uma rápida solução de problemas. Foi relatado que existem pouquíssimos ruídos na comunicação formal na área. Todavia, o grande entrave na comunicação é externo, advindo de outras áreas. Isto significa que outras secretarias e departamentos da organização, quando fazem solicitações a essa secretaria, não fornecem informações claras e objetivas sobre suas demandas, bem como não utilizam os meios adequados para acessar a área, ocasionando dificuldade de compreensão e entrega de resultados incompatíveis com o desejado.

No que tange à autonomia, foi enfatizada a forte participação dos servidores nas decisões tomadas. Na verdade, foi apontado que os mesmos possuem um alto grau de responsabilidade, isto significa, por exemplo, 
que os servidores podem negociar diretamente com o cliente, sem necessariamente pedir autorização ou esperar uma decisão de seus supervisores. A criatividade é um aspecto bastante valorizado pelos gestores; portanto, possuem um alto grau de autonomia na proposição de soluções, para não inibir o processo criativo.

\section{Bem-estar no trabalho}

O conceito de bem-estar para os participantes entrevistados tem forte relação com aspectos físicos, e nem tanto afetivos, como na definição constitutiva utilizada neste trabalho. De imediato, o conceito de bem-estar no trabalho foi associado com conforto físico, isto é, com a promoção de uma estrutura física confortável e ergonômica por parte da organização. Após alguns argumentos, chegou-se ao consenso de que o clima de descontração é um fator que promove o bem-estar no trabalho, tendo em vista que essa descontração contribui para o alívio do estresse, e este, por sua vez, é tido como oposto de bem-estar. Um ambiente silencioso, ou com o mínimo de barulho possível, também é um promotor de vivências de bem-estar no trabalho.

\section{Satisfação no trabalho}

O conceito de satisfação no trabalho está relacionado a diversos aspectos e se assemelhou muito com a proposta de Siqueira (2008), que a define como o grau de contentamento com a chefia, com os colegas, com o salário, com promoções e com o trabalho realizado. Para os participantes, satisfação tem a ver com o salário, com a autonomia, com a chefia, com os colegas e com a ascensão funcional. É de suma importância ressaltar que esses resultados não foram induzidos e em nenhum momento foi apresentada a definição de Siqueira (2008). Dessa forma, a obtenção desse conceito de satisfação no trabalho demonstra a importância e a relevância da definição de Siqueira (2008) para os estudos organizacionais.

Diante do exposto, a satisfação com salário está relacionada à percepção de justiça do valor recebido, isto é, se o mesmo é compatível com a atividade desempenhada. A satisfação com a autonomia tem a ver com a liberdade de criação e com o processo decisório relativo às atividades realizadas pelo servidor. A satisfação com a chefia está relacionada ao relacionamento "portas abertas", em que o servidor tem liberdade para conversar abertamente com o chefe, e também tem relação com o apoio que o gestor dá aos projetos desenvolvidos pelo servidor. A satisfação com os colegas é advinda de uma boa interação e de um bom relacionamento interpessoal. A satisfação com a ascensão funcional significa que o servidor teve seu trabalho reconhecido e que possui as competências necessárias para assumir novas responsabilidades.

\section{Bem-estar no trabalho e desempenho individual no trabalho}

Em um segundo momento, as questões foram direcionadas às relações entre as variáveis. Sobre a relação entre bem-estar e desempenho, inicialmente a discussão caminhou para o fato de que aspectos de bem-estar contribuem para um bom desempenho. Consoante com entendimento do que é bem-estar, os participantes concordaram que é muito melhor produzir em um ambiente leve, tranquilo, sossegado. E que o clima de descontração da área ajuda para obtenção de um melhor desempenho. Os aspectos negativos também foram mencionados, como o fato de estar preocupado ou ansioso não favorece o desempenho.

Apesar de o consenso inicial e dos comentários convergirem para os pontos apresentados acima, um dos participantes explicou que essa relação entre bem-estar contribui para o desempenho, mas não é um fator essencial para a obtenção de melhores desempenhos; em outras palavras, é possível obter desempenhos de excelência mesmo sem vivenciar situações de bem-estar. Esse apontamento gerou certo desconforto nos demais participantes, mas, após a discussão, houve um consenso de que bem-estar não possui relação direta com desempenho. $\mathrm{O}$ argumento apresentado foi de que aspectos como a pressão do chefe e do cliente que normalmente geram sensações de mal-estar, como o estresse, muitas vezes, contribuem para o incremento do desempenho. Diversas experiências foram relatadas a fim de exemplificar essa afirmativa, como, por exemplo, em períodos nos quais são desenvolvidos projetos que possuem prazo determinado, e tanto os gestores quantos os clientes pressionam a equipe para que entreguem os resultados no tempo previsto. 


\section{Bem-estar no trabalho e estrutura organizacional}

Quando questionados de que forma a estrutura organizacional contribuía para o bem-estar no trabalho, a tônica da discussão girou em torno da informalidade da área. Como se trata de uma secretaria de comunicação, é de suma importância que a estrutura seja adaptada de modo a não bloquear a criatividade. Os participantes da seção de programação visual foram os mais animados para discutir essa relação. Para eles, é fundamental que exista autonomia no desempenho das funções e que a comunicação entre a equipe e os superiores flua sem entraves. Essas condições contribuem para a liberdade de criação, e, consequentemente, são excelentes para a criatividade. Com o ambiente favorável para a geração de ideias, os funcionários ficam mais contentes por trabalharem com o que gostam e empolgados por terem a oportunidade de mostrarem suas competências.

\section{Satisfação no trabalho e desempenho individual no trabalho}

Ao tratar da relação entre satisfação no trabalho e desempenho, os participantes relataram que, quando satisfeitos com a atividade que realizam, se sentem mais motivados, têm mais disposição para ir trabalhar, e o trabalho flui. E, se o trabalho flui, a consequência direta é melhora no desempenho. Além disso, é percebido um melhor desempenho, quando há uma maior interação com os colegas, isso porque a troca de ideias e a possibilidade de conhecer diferentes pontos de vista, devido às diferentes formações, aumenta a satisfação com o trabalho.

Um fator que entrou em evidência foi a questão da satisfação com o salário e a remuneração indireta. Os servidores com mais tempo de casa explicaram que o bom desempenho pode ser justificado pelo fato de que o contexto de trabalho e os benefícios oferecidos são bastante atrativos e compensam o salário não tão interessante. A satisfação com esses aspectos é tamanha que os participantes disseram que essa é uma das principais motivações para trabalhar e para desempenhar suas atividades e tarefas. Por sua vez, os servidores mais novos não têm aumento salarial há oito anos, e essa insatisfação tem impactado no desempenho desses indivíduos.

\section{Satisfação no trabalho e estrutura organizacional}

Como tratado em outros momentos, a liberdade de criação é um fator crucial para os servidores dessa área. Os participantes alegaram que no processo de criação é permitido que utilizem seu próprio material e recursos do seu portfólio. Eles apontaram que as oportunidades dadas pela organização, especialmente em relação ao processo de tomada de decisão no desenvolvimento de projetos. Quando questionados sobre a satisfação com a ascensão funcional, todos em uníssono demonstraram estar completamente satisfeitos; nos termos utilizados pelos participantes, eles estão $100 \%$ satisfeitos. Isso se justifica, por se tratar de uma organização pública, que os critérios de ascensão para níveis hierárquicos superiores estão bem claros.

Em relação à chefia, os servidores demonstraram um bom nível de satisfação, pois a organização é adepta da política de "portas abertas", na qual os funcionários podem acessar seu superior diretamente sem entraves, ou sem ter que passar por outras pessoas para viabilizar o acesso. Por outro lado, foi apontado um aspecto negativo da organização em relação a questões de chefia. A gestão das secretarias é um cargo político, isso significa que os gestores dessas mudam a cada ciclo político. Esse é um aspecto dificultador de desempenho e, principalmente, gerador de insatisfação, pois os gestores das coordenações atendem às necessidades de suas áreas, mas não conseguem levar seus projetos adiante por falta de interesse ou de alinhamento com os interesses políticos da organização. Também existem casos em que projetos em andamento são encerrados ao iniciar uma nova gestão. Somada a essas dificuldades, a política de "portas abertas" não se estende a esse nível de gestores, tornando-os praticamente inacessíveis.

\section{Estrutura organizacional e desempenho individual no trabalho}

Diversos aspectos da estrutura organizacional foram apontados com facilitadores ou dificultadores do desempenho no trabalho. Uma questão bastante discutida foi a comunicação. Quando questionados sobre a 
comunicação dentro de suas áreas, todos os participantes afirmaram que a comunicação interna flui bem, de modo que ruídos ou falhas não comprometam a realização de suas tarefas. Todavia, a relação com outras áreas são mais delicadas, especialmente com aquelas que não pertencem à secretaria. Comentou-se que determinadas áreas exageram nos contatos telefônicos e no envio de e-mails, tornando-se inconvenientes e, muitas vezes, interrompendo o desenvolvimento de uma atividade.

Como dito em outros momentos, a autonomia é um fator bastante ressaltado pelos participantes e é de suma importância para um bom desempenho, isso porque as decisões podem ser tomadas imediatamente, sem que o funcionário tenha que recorrer a um superior hierárquico. Depreende-se, portanto, que os servidores possuem uma participação forte no processo decisório, e que isso contribui para que as atividades sejam cumpridas em tempo hábil e que os objetivos e metas sejam alcançados.

No entendimento dos participantes, a estrutura organizacional compreende aspectos físicos e materiais; dessa forma, esses fatores também foram contemplados na relação com desempenho. A qualidade dos equipamentos foi apontada como um fator positivo para o desempenho das tarefas; por sua vez, a rede de internet da organização é ruim, e o sistema interno da organização bloqueia arquivos no backup e não permite acesso a determinados recursos na rede ou de softwares necessários para o trabalho, tornando-se obstáculos para o desenvolvimento das atividades. Ressalta-se que, apesar desses entraves, a equipe de informática é bastante efetiva no atendimento das solicitações para a solução de problemas técnicos.

\section{Discussão}

Segundo os participantes, a autonomia foi o aspecto da estrutura mais citado e mais defendido como promotor de bem-estar e satisfação no trabalho e como facilitador do desempenho individual. A liberdade de criação e a rápida solução de problemas foram as razões apontadas como os benefícios decorrentes da autonomia no processo decisório. A literatura também reforça o papel da autonomia como preditora de desempenho no trabalho (LANGFRED, 2005; LANGFRED e MOYE, 2004; LEACH, WALL e ROGELBERG, 2005; MORGESON, DELANEY-KLINGER e HEMINGWAY, 2005; OZER, 2011; STEWART, 2006). Destaca-se o trabalho de Ozer (2011), que estudou o efeito moderador da autonomia na relação entre cidadania organizacional e desempenho no trabalho. Diante dessas evidências, depreende-se que a percepção dos trabalhadores se alinha com as pesquisas realizadas anteriormente.

Esse achado suscita duas questões: que razões poderiam ser atribuídas pela escolha da autonomia como fator preponderante da estrutura para ser o componente mais estudado com desempenho no trabalho? E por que outros componentes da estrutura não recebem a mesma atenção em pesquisas que analisam preditores do desempenho no trabalho? Observando os resultados do grupo, percebe-se que outros aspectos da estrutura são relevantes, como a estrutura informal.

Autores como Oliveira (2006) e Vasconcellos e Hemsley (2002) apontam que a estrutura organizacional pode ser dividida em duas partes: a formal e a informal. A formal é aquela que foi planejada e que, na maioria das vezes, tem como principal meio de representação o organograma. A informal envolve aspectos resultantes das interações sociais dos membros da organização. $\mathrm{O}$ interessante é que, quando se fala em componentes da estrutura organizacional, a impressão que se tem é que as dimensões estão relacionadas à estrutura formal. E talvez essa seja uma das razões pelas quais os estudos envolvendo aspectos informais não sejam tão disseminados.

Independentemente do conhecimento da produção existente, o fato que é o resultado obtido aponta para a relevância dos aspectos informais para o desempenho do indivíduo na organização. Essa evidência abre precedentes para investigações futuras sobre o tema e, principalmente, para o aprofundamento do estudo da relação entre a "estrutura informal" e o desempenho, contribuindo para que sejam desenvolvidas práticas para a gestão da estrutura informal de modo que contribua para o alcance dos objetivos organizacionais. 
No trabalho de Kaplan, Bradley, Luchman et al. (2009) foram empreendidas diversas meta-análises que apontaram para a relação preditiva dos afetos positivos e dos afetos negativos com desempenho nas tarefas. Para os autores, essa constatação deve direcionar as ações das organizações para promover o bem-estar, de modo que devem ser minimizadas as emoções negativas, como estresse e ansiedade, e incentivadas as positivas, como excitação e entusiasmo. A significância dessa evidência confronta a percepção dos participantes deste estudo, que acreditavam na relação entre bem-estar e desempenho, mas que essa relação não era direta, nem tampouco essencial.

Observando esses resultados, é possível entender porque a estrutura organizacional contribuiu para reforçar a relação entre bem-estar e desempenho. Os participantes apontaram que a informalidade e a autonomia são fatores bastante importantes para gerar bem-estar no trabalho. Esses resultados explicam que os aspectos informais e autonomia propiciam bem-estar no trabalho, bem como também são favoráveis para melhores desempenhos. Considerando que os aspectos informalidade e autonomia propiciam bem-estar, pode-se entender que eles aumentam os afetos positivos e diminuem os afetos negativos. Em relação à satisfação e com base na definição utilizada neste estudo, os participantes atribuíram maior importância para os fatores "satisfação com os colegas de trabalho" e "satisfação com a natureza do trabalho". Sintetizando os achados, a evidência obtida aqui aponta para o desenvolvimento de estudos que contribuam para o desenvolvimento de uma teoria que sustente empiricamente a relação entre satisfação no trabalho, bem-estar no trabalho, desempenho individual no trabalho e estrutura organizacional. E, dessa forma, possa fundamentar a tão disseminada hipótese "trabalhador feliz, produtivo".

\section{Conclusão}

Existem outros aspectos envolvidos no ato de trabalhar, como qualidade de vida, preocupação social e bemestar, que podem beneficiar ou prejudicar a empresa. Dessa forma, enxergar um funcionário como um mero recurso pode representar abrir mão de uma vantagem competitiva e profissionais talentosos, que, na primeira oportunidade, migrarão para um concorrente que ofereça melhores condições de trabalho. O fato é que as organizações não percebem com clareza o impacto que a estrutura pode exercer no desempenho do trabalhador, e mais, a relevância do bem-estar e da satisfação na realização do trabalho. Em se tratando de estrutura organizacional, especialmente, este trabalho contribui significativamente para a inclusão desse construto em pesquisas voltadas para variáveis de nível micro, demonstrando o impacto que a estrutura exerce no comportamento dos indivíduos.

E a percepção dos trabalhadores, qual sua relevância teórica? Esta pesquisa identificou que os participantes julgam que a satisfação é muito importante para a obtenção de melhores índices de desempenho. Mas será que essa percepção é em decorrência de um senso comum disseminado ao longo dos anos de que a satisfação no trabalho impacta fortemente no desempenho? Ou seria realmente a crença desses trabalhadores com base em sua vivência diária? Logicamente, não será fácil encontrar a resposta para essas questões, mas a reflexão feita em cima delas possibilitará a geração de insights para o desenvolvimento de pesquisas sobre o tema.

Espera-se que este trabalho possa ser um norte para o avanço de pesquisas futuras voltadas para as variáveis analisadas aqui. Ressalta-se uma preocupação com o desenvolvimento de mais estudos que envolvam a estrutura organizacional, dada a sua relevância para pesquisadores da área de administração, pois as organizações são o seu objeto de estudo. A identificação de uma série de convergências nas respostas permite levantar algumas hipóteses para estudos quantitativos futuros, a saber:

- O clima organizacional poderá aumentar positivamente a relação entre bem-estar no trabalho e desempenho individual no trabalho;

- a estrutura física estará positivamente associada com bem-estar no trabalho;

- a estrutura física estará positivamente associada com o desempenho individual no trabalho; 
- a autonomia poderá aumentar positivamente a relação entre satisfação no trabalho e desempenho individual no trabalho;

- a estrutura informal estará positivamente associada com bem-estar no trabalho.

Essas são sugestões de hipóteses, é claro, e podem servir como base para o desenvolvimento de pesquisas futuras. Como também podem existir outras hipóteses nas entrelinhas dos resultados. O intuito é, portanto, incentivar que trabalhos sejam empreendidos para promover o avanço do tema, ampliar as discussões sobre o assunto no contexto organizacional e consolidar a teoria sobre cada uma das variáveis.

\section{Referências}

ANSEEL, F.; LIEVENS, F. The long-term impact of feedback environment on job satisfaction: A field study in a Belgian context. Applied Psychology, v. 56, n. 2, p. 254-266, 2007.

ARYEE, S.; CHEN, Z. X.; BUDHWAR, P. S. Exchange fairness and employee performance: An examination of the relationship between organizational politics and procedural justice. Organizational Behavior and Human Decision Processes, v. 94, n. 1, p. 1-14, 2004.

BARDIN, L. Análise de conteúdo. Lisboa: Edições 70, 2009.

BATES, R. A. Measuring performance improvement. Advances in Developing Human Resources, v.1, n.1, p. 47-67, 1999.

BOWLING, N. A. Is the job satisfaction - job performance relationship spurious? A meta-analytic examination. Journal of Vocational Behavior, v. 71, n. 2, p. 167-185, 2007.

BRETZ Jr., R. D.; MILKOVICH, G. T.; READ, W. The current state of performance appraisal research and practice: Concerns, directions, implications. Journal of Management, v. 18, n. 2, p. 321-352, 1992.

BRIEF, A. P.; WEISS, H. M. Organizational behavior: Affect at work. Annual Review of Psychology, v. 53, p. 279$307,2002$.

BURREL, G.; MORGAN, G. Sociological Paradigms and Organizational Analysis. Aldershot, UK: Ashgate Publishing Co, 2001.

CAMPBELL, J. P. Modeling the performance prediction problem in industrial and organizational psychology. In: DUNNETTE, M. D.; HOUGH, L. M. (Eds.).Handbook of industrial and organizational psychology, 1. Palo Alto: Consulting Psychologists Press, Inc., 1990. 687-732 p.

CAMPBELL, S. L.; FOWLES, E. R.; WEBER, B. J. Organizational Structure and Job Satisfaction in Public Health Nursing. Public Health Nursing, v. 21, n. 6, p. 564-571, 2004.

CHANG, S. C.; LEE, M. S. Relationships among personality traits, job characteristics, job satisfaction and organizational commitment - an empirical study in Taywan. The Business Review, v.6, n.1, p.201-207, 2006.

CHASE, R. B.; JACOBS, F. R.; AQUILANO, N. J. Administração da produção para a vantagem competitiva. Porto Alegre: Bookman, 2006.

COELHO Jr., F. A. Suporte à aprendizagem, satisfação no trabalho e desempenho: Um estudo multinível. 2009. 301f. Tese (Doutorado em Psicologia) - Instituto de Psicologia, Universidade de Brasília, Brasília.

COELHO Jr., F. A.; BORGES-ANDRADE, J. E. Efeitos de variáveis individuais e contextuais sobre desempenho individual no trabalho. Estudos de Psicologia, v. 16, n. 2, p. 111-120, 2011. 
COELHO Jr., F. A. et al. O comportamento organizacional como campo científico: uma análise crítica. In: Encontro da Associação Nacional de Pós-Graduação e Pesquisa em Administração, 36. Rio de Janeiro. Anais... Rio de Janeiro: ANPAD, 2012.

CROPANZANO, R.; RUPP, D. E.; BYRNE, Z. S. The relationship of emotional exhaustion to work attitudes, job performance, and organizational citizenship behaviors. Journal of Applied Psychology, v. 88, n. 1, p. 160-169, 2003.

DEADRICK, D. L.; GARDNER, D. G. Maximal and typical measures of job performance: An analysis of performance variability over time. Human Resource Management Review, v. 18, p. 133-145, 2008.

DEJOURS, C. Cadernos de TTO - Volume 2: Avaliação do trabalho submetida à prova do real. São Paulo: Ed. Blucher, 2008.

DESSEN, M. C.; PAZ, M. G. T. Bem-estar pessoal nas organizações: O impacto de configurações de poder e características de personalidade. Psicologia: Teoria e Pesquisa, v. 26, n. 3, p. 549-556, 2010.

DOEST, L.; DE JONGE, J. Testing causal models of job characteristics and employee well-being: A replication study using cross-lagged structural equation modeling. Journal of Occupational and Organizational Psychology, v. 79, n. 3, p. 499-507, 2006.

EDWARDS, B. D. et al. Relationships between facets of job satisfaction and task and contextual performance. Applied Psychology, v. 57, n. 3, p. 441-465, 2008.

FISCHER, C. D. Why do lay people believe that satisfaction and performance are correlated? Possible sources of commonsense theory. Journal of Organizational Behavior, v.24, n.6, p. 753-777, 2003.

FLICK, U. Introdução à pesquisa qualitativa. São Paulo: Artmed, 2009.

GONÇALVES, E. L. Estrutura organizacional do hospital moderno. Revista de Administração de Empresas, v. 38, n. 1, p. 80-90, 1998.

GONDIM, S. M. G.; SIQUEIRA, M. M. M. Emoções e afetos no trabalho. In: ZANELLI, J. C.; BORGES-ANDRADE, J. E.; BASTOS, A. V. B. (Eds.). Psicologia, Organizações e Trabalho no Brasil. Porto Alegre: Artmed, 2004. $207-$ $236 \mathrm{p}$.

GORIS, J. R.; VAUGHT, B. C.;PETIT Jr., J. D. Effects of communication direction on job performance and satisfaction: a moderated regression analysis. Journal of Business Communication, v.37, n.4, p. 348-368, 2000.

GOUVEIA, V. V. et al. Escala de bem-estar afetivo no trabalho (JAWS): Evidências de validade fatorial e consistência interna. Psicologia: Reflexão e Crítica, v.21, n.3, p. 464-473, 2008.

GRØNKJÆR, M. et al. Analysing group interaction in focus group research: Impact on content and the role of the moderator. Qualitative Studies, v.2, n.1, p. 16-30, 2011.

HALBESLEBEN, J. R. B.; BOWLER, W. M. Emotional exhaustion and job performance: The mediating role of motivation. Journal of Applied Psychology, v. 92, n. 1, p. 93-106, 2007.

JANSSEN, O.; LAM, C. K.; HUANG, X. Emotional exhaustion and job performance: The moderating roles of distributive justice and positive affect. Journal of Organizational Behavior, v. 31, n. 6, p. 787-809, 2010.

KAPLAN, S. et al. On the role of positive and negative affectivity in job performance: A meta-analytic investigation. Journal of Applied Psychology, v. 94, n. 1, p. 162-176, 2009.

KIRSCHENBAUM, A.; OIGENBLICK, L.; GOLDBERG, A. I. Wellbeing, work environment and work accidents. Social Science and Medicine, v.50, n.5, p. 631-639, 2000.

LADEIRA, W. J.; SONZA, I. B.; BERTE, R. S. Antecedentes da satisfação no setor público: Um estudo de caso na prefeitura de Santa Maria (RS). Rev. Adm. Pública, v. 46, n. 1, p. 71-91, 2012. 
LANGFRED, C. W. Autonomy and performance in teams: The multilevel moderating effect of task interdependence. Journal of Management, v. 31, n. 4, p. 513-529, 2005.

LANGFRED, C. W.; MOYE, N. A. Effects of task autonomy on performance: An extended model considering motivational, informational, and structural mechanisms. Journal of Applied Psychology, v. 89, n. 6, p. 934-945, 2004.

LASCHINGER, H. K. S. et al. A longitudinal analysis of the impact of workplace empowerment on work satisfaction. Journal of Organizational Behavior, v. 25, n. 4, p. 527-545, 2004.

LEACH, D. J.; WALL, T. D.; ROGELBERG, S. G. Team autonomy, performance, and member job strain: Uncovering the teamwork KSA link. Applied Psychology, v. 54, n. 1, p. 1-24, 2005.

LIN, W.; WANG, L.; CHEN, S. Abusive supervision and employee well-being: the moderating effect of power distance orientation. Applied Psychology,v.62, n.2, p. 308-329, 2013.

MIERLO, H. V. et al. A multi-level mediation model of the relationships between team autonomy, individual task design and psychological well-being. Journal of Occupational and Organizational Psychology, v. 80, n. 4, p. 647$664,2007$.

MORGESON, F. P.; DELANEY-KLINGER, K.; HEMINGWAY, M. A. The importance of job autonomy, cognitive ability, and job-related skill for predicting role breadth and job performance. Journal of Applied Psychology, v. 90, n. 2, p. 399-406, 2005.

NETEMEYER, R. G.; MAXHAM III, J. G.; LICHTENSTEIN, D. R. Store manager performance and satisfaction: Effects on store employee performance and satisfaction, store customer satisfaction, and store customer spending growth. Journal of Applied Psychology,v.95, n.3, p. 530-545, 2010.

OLIVARES, J. E. L. Análise da estrutura organizacional em rede e suas negociações no contexto de alianças estratégicas. 2003. 230 f. Tese (Doutorado em Administração), Universidade de São Paulo, São Paulo.

OLIVEIRA, D. P. R. Sistemas, organização e métodos: Uma abordagem gerencial. São Paulo: Editora Atlas, 2006.

OLIVEIRA, M.; FREITAS, H. M. R. Focus Group - pesquisa qualitativa: resgatando a teoria, instrumentalizando o seu planejamento. Revista de Administração, v.33, n.3, p. 83-91, 1998.

OLIVEIRA-CASTRO, G. A.; LIMA, G. B. C.; VEIGA, M. R. M. Implantação de um sistema de avaliação de desempenho: Métodos e estratégias. Revista de Administração, v. 31, n. 3, p. 38-52, 1996.

OZER, M. A moderated mediation model of the relationship between organizational citizenship behaviors and job performance. Journal of Applied Psychology, v. 96, n. 6, p. 1328-1336, 2011.

PARKER, S. K.; TURNER, N. Work design and individual work performance: Research findings and an agenda for future inquiry. In: SONNENTAG, S. (Ed.). Psychological Management of Individual Performance. Great Britain: John Wiley \& Sons, 2002. 69-95 p.

PASCHOAL, T. Bem-estar no trabalho: Relações com suporte organizacional, prioridades axiológicas e oportunidade de alcance de valores pessoais no trabalho. 2008. 180f. Tese (Doutorado em Psicologia) - Instituto de Psicologia, Universidade de Brasília, Brasília.

PASCHOAL, T.; TAMAYO, A. Construção e Validação da Escala de Bem-Estar no Trabalho. Avaliação Psicológica, v. 7, n. 1, p. 11-22, 2008.

PASCHOAL, T. et al. Well-being at work: Scenario of Brazilian studies published in the first decade of the millennium. Revista Encontros Científicos: Tourism and Management Studies, v. 2, p. 383-395, 2013.

REGO, A. Empregados felizes são mais produtivos? Revista de Estudos Politécnicos, v. 7, n. 12, p. 215-233, 2009.

SAMPIERI, R. H.; COLlADO, C. F.; LUCIO, P. B. Metodologia de Pesquisa. São Paulo: McGraw Hill, 2006. 
SCHLEICHER, D. J.; WATT, J. D.; GREGURAS, G. J. Reexamining the job satisfaction-performance relationship: The complexity of attitudes. Journal of Applied Psychology, v. 89, n. 1, p. 165-177, 2004.

SIQUEIRA, M. M. M. Medidas do comportamento organizacional. Estudos de psicologia, v. 7, p. 11-18, 2002.

SIQUEIRA, M. M. M. Satisfação no trabalho. In: SIQUEIRA, M. M. M. (Ed.). Medidas do Comportamento Organizacional. Porto Alegre: Artmed, 2008. 265-274 p.

SIQUEIRA, M. M. M.; AMARAL, D. J. Relações entre estrutura organizacional e bem-estar psicológico. Revista Eletrônica de Administração (REA), v. 7, p. 1-16, 2006.

SONNENTAG, S. Performance, well-being and self-regulation. In: SONNENTAG, S. (Ed.). Psychological Management of Individual Performance. Great Britain: John Wiley \& Sons, 2002. 405-425 p.

SONNENTAG, S.; FRESE, M. Performance concepts and performance theory. In: SONNENTAG, S. (Ed.). Psychological Management of Individual Performance. Great Britain: John Wiley \& Sons, 2002. 3-27 p.

STEWART, G. L. A meta-analytic review of relationships between team design features and team performance. Journal of Management, v. 32, n. 1, p. 29-55, 2006.

SY, T.; TRAM, S.; O'HARA, L. A. Relation of employee and manager emotional intelligence to job satisfaction and performance. Journal of Vocational Behavior, v. 68, n. 3, p. 474-489, 2006.

TSANG, H. W. H.; WONG, A. Scale CV-IJSS for people with mental illness development and validation of the Chinese version of Indiana Job Satisfaction. International Journal of Social Psychiatry, v. 51, n. 2, p. 177-191, 2005.

VAN HORN, J. E. et al. The structure of occupational well-being: A study among Dutch teachers. Journal of Occupational and Organizational Psychology, v. 77, n. 3, p. 365-375, 2004.

VASCONCELLOS, E.; HEMSLEY, J. R. Estrutura das organizações: Estrutura tradicional, estrutura para inovação, estrutura matricial. São Paulo: Pioneira, 2002.

WARR, P. Work, happiness and unhappiness. New Jersey: Lawrence Erlbaum Associates, 2007.

WORRELL, T. G.; SKAGGS, G. E.; BROWN, M. B. A comparison of two measures of school psychologists' job satisfaction. Canadian Journal of School Psychology, v. 21, p. 47-58, 2006.

WRIGHT, T. A.; CROPANZANO, R. Psychological well-being and job satisfaction as predictors of job performance. Journal of Occupational Health Psychology, v. 5, n. 1, p. 84-94, 2000.

WRIGHT, T. A.; HUANG, C. The many benefits of employee well-being in organizational research. Journal of Organizational Behavior, v. 33, n. 8, p. 1188-1192, 2012. 